\title{
Case Study of Anomaly Detection and Quality Control of Energy Efficiency and Hygrothermal Comfort in Buildings
}

\author{
Carlos Eiras-Franco ${ }^{1}{ }^{\mathrm{a}}{ }^{\mathrm{a}}$, Miguel Flores ${ }^{2}{ }^{\mathrm{b}} \mathrm{b}$, Verónica Bolón-Canedo ${ }^{1}{ }^{\mathrm{c}}{ }^{\mathrm{c}}$, Sonia Zaragoza ${ }^{3,4}$,

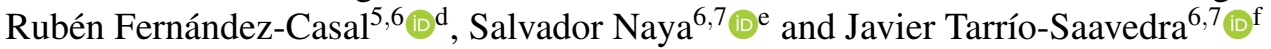 \\ ${ }^{1}$ LIDIA Group, Department of Computer Science, CITIC, Universidade da Coruña, Campus de Elviña, A Coruña, Spain \\ ${ }^{2}$ Department of Mathematics, Escuela Politécnica Nacional, Quito, Ecuador \\ ${ }^{3}$ PROTERM Group, Department of Naval and Industrial Engineering, Escola Politécnica Superior, \\ Universidade da Coruña, Mendizábal s/n, Ferrol, Spain \\ ${ }^{4}$ ¿qus company, Oleiros, Spain \\ ${ }^{5}$ MODES Group, Department of Mathematics, Facultade de Informática, Universidade da Coruña, \\ Campus de Elviña, A Coruña, Spain \\ ${ }^{6}$ Centro de Investigación TIC (CITIC), Universidade da Coruña, Campus de Elviña, A Coruña, Spain \\ ${ }^{7}$ MODES Group, Department of Mathematics, Escola Politécnica Superior, Universidade da Coruña, \\ Mendizábal s/n, Ferrol, Spain
}

Keywords: $\quad$ Statistical Quality Control, Anomaly Detection, Feature Selection, Energy Efficiency, HVAC, Industry 4.0, LOCI, ReliefF, Functional Data Analysis.

\begin{abstract}
The aim of this work is to propose different statistical and machine learning methodologies for identifying anomalies and control the quality of energy efficiency and hygrothermal comfort in buildings. Companies focused on energy sector for buildings are interested on statistical and machine learning tools to automate the control of energy consumption and ensure quality of Heat Ventilation and Air Conditioning (HVAC) installations. Consequently, a methodology based on the application of the Local Correlation Integral (LOCI) anomaly detection technique has been proposed. In addition, the most critical variables for anomaly detection are identified by using ReliefF method. Once vectors of critical variables are obtained, multivariate and univariate control charts can be applied to control the quality of HVAC installations (consumption, thermal comfort). In order to test the proposed methodology, the companies involved in this project have provided the case study of a store of a clothing brand located in a shopping center in Panama. It is important to note that this is a controlled case study for which all the anomalies have been previously identified by maintenance personnel. Moreover, as an alternatively solution, in addition to machine learning and multivariate techniques, new nonparametric control charts for functional data based on data depth have been proposed and applied to curves of daily energy consumption in HVAC.
\end{abstract}

\section{INTRODUCTION}

The recent advances in the framework of Industry 4.0 allow the companies to monitor the processes that define products and services (Naya, 2017) continuously with respect to time. The improvements correspond-

\footnotetext{
a (iD) https://orcid.org/0000-0001-6322-7593

b (iD) https://orcid.org/0000-0002-7742-1247

c (iD https://orcid.org/0000-0002-0524-6427

d (iD https://orcid.org/0000-0002-5785-3739

e (iD https://orcid.org/0000-0003-4931-9859

f(D) https://orcid.org/0000-0002-9584-127X
}

ing to sensoring have lead a high rising in volume and variety of data, now easily available in a remote way through web applications. This new paradigm of data make difficult to control manually the quality of processes. Thus, statistical and machine learning techniques that automate the procedures of anomaly detection and quality control of products and services are increasingly needed (Lee et al., 2014). Specifically, the companies of building energy efficiency sector have recently developed energy web platforms that require the implementation of statistical tools to automate the anomaly detection, the predictive maintenance, and the quality control of building installa- 
tions (Barbeito et al., 2017; Flores et al., 2018). That is the case of web platform developed by $\Sigma$ qus company, that provides the real case study described in this work. It consist on the energy deficiency control of the Heating, Ventilation and Air Conditioning (HVAC) installation of a clothing store placed in Panama City, from the data provided by $\Sigma$ qus web platform.

The statistical models used to detect anomalies could be classified in two main groups. On one hand, those based on the application of supervised classification techniques (Francisco-Fernández et al., 2012; Mallik et al., 2011; Bolon-Canedo et al., 2011; BolonCanedo et al., 2017) and, on the other hand, the control charts, in the framework of statistical quality control (Barbeito et al., 2017). Control charts, either for scalar or multivariate cases, have been profusely used in all the sectors of industry (Montgomery, 2007). Broadly speaking, control charts estimate the range of normal performance of a process, i.e. they provide information about the studied process is in control. If the process is not under control, this could be related with the presence of an anomaly in the process. When using control charts, training a model considering of all the types of anomalies is not necessary. This is an advantage with respect to machine learning methods based on supervised classification. This goal can be extrapolated to those cases in which the quality of a process is defined by the relation between two variables by using the profile control charts. These charts are used when the process is defined by curves depending on time or frequency, among others continuous variables (Woodall, 2007), in fact they can be studied from the Functional Data Analysis (FDA) approach, a branch of statistics that includes all those techniques that can be applied when data are curves (infinite dimension data) (Ferraty and Vieu, 2006; Francisco-Fernández et al., 2012). In this work, a FDA methodology based on functional data depth (López-Pintado and Romo, 2009) combined with nonparametric control charts based on ranks (Liu, 1995) is proposed.

This study is organized as follows. The Section 2 describes the energy efficiency case study, and its corresponding dataset composed of critical to quality variables for building energy efficiency. In Section 3, the machine learning techniques applied to detect anomalies and to extract the relevant features that help to identify them are briefly introduced. Section 4 accounts for the FDA control chart approach introduction and description. The results obtained from the application of the statistical and machine learning methodologies are presented in Section 5, whereas the Section 6 includes the final remarks.

\section{CASE STUDY: DETECTION OF ANOMALIES DEALING WITH THE ENERGY EFFICIENCY OF HVAC INSTALLATIONS}

A clothing store located in a commercial center of Panama City is studied by continuously monitoring using the $\Sigma$ qus web platform. Overall 16 critical to quality variables are measured, including indoor temperatures, energy consumption, energy consumption in HVAC, relative humidity, $\mathrm{CO}_{2}$ amount, and temperatures of impulsion and return corresponding to the chillers of the different areas of the store (see Figure 1).

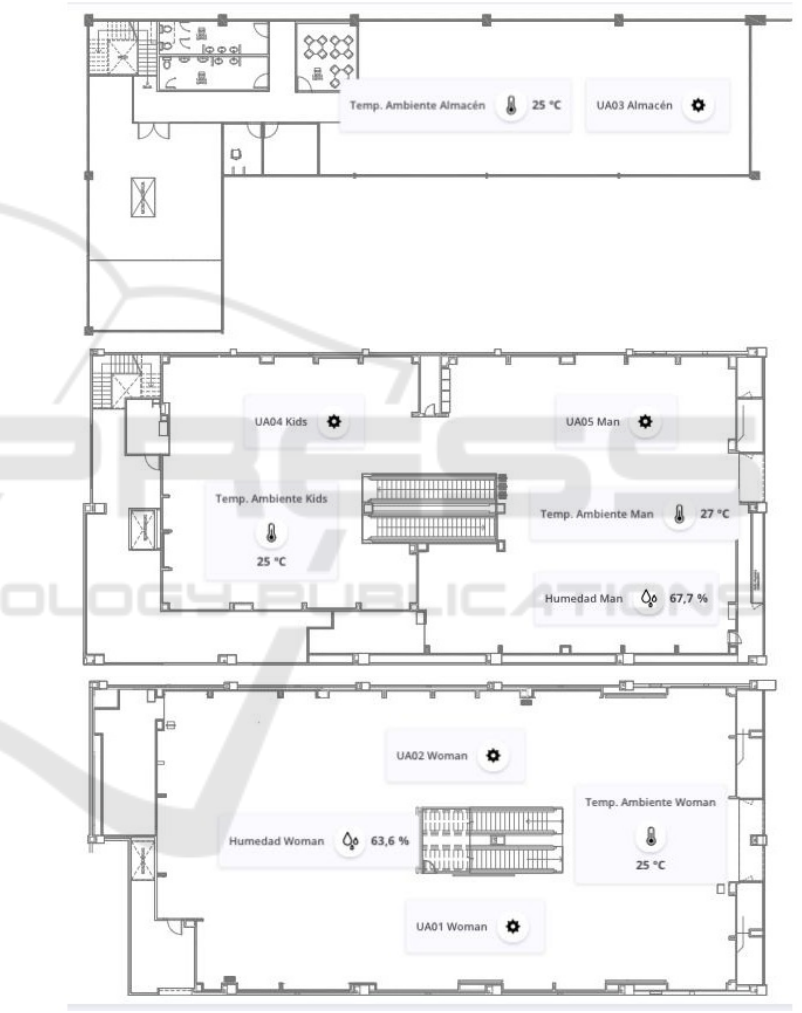

Figure 1: Plan of the study case store placed in Panama City.

Hourly measurements are obtained from August 1 2017 to October 312018 . The HVAC installation of the store begins to run at 9:00 or 10:00 in the morning. At start-up, a peak in the energy consumption occurs due to the characteristics of the HVAC installation. From 12:00, consumption remains relatively constant until 20:00, 21:00 pm or 22:00 pm, when the store closes. The shut-down takes about 1 or 2 hours, with consumption falling at a constant rate of change. The resulting data can be considered functional data and thus FDA techniques can be applied. It is also impor- 
tant to note that this case study is a controlled study in which the anomalies and their assignable causes have been previously detected for the maintenance staff.

In the following lines, some of the anomalies identified by the maintenance staff are described. Thus, on September 11 there was a decrease in air conditioning consumption towards the middle of the day. On September 21, 22 and 30 the shopping center was closed, so there was no consumption and temperatures were high. On September 27, maintenance tests were carried out at the store facilities. On September 29, the store HVAC installation was stopped one hour earlier than usual. As of September 19, the air conditioning is turned off half an hour before, that is, there is a regulation change in the HVAC system. At the middle of October, there is a leak in the air conditioning circuit. From that moment, energy consumption began to rise. Moreover, on November 1, repairing activities were made. Consequently, the consumption decreased and, in addition, the star-up consumption peak was prevented. Between November 17 and 20 the consumption returned to increase. Apart from the anomalies above mentioned, many others have been detected and used to train and evaluate the proposed models.

\section{STATISTICAL AND MACHINE LEARNING METHODOLOGIES}

\subsection{Machine Learning Methodology}

The case study data are vectors composed of 18 variables (16 variables in addition to the date and time) that correspond to the measurements made by 14 sensors and 2 meters every 5 minutes. We have transformed these measurements into daily vectors in order to perform the analysis of anomalies at the level of full days. For this purpose, the 288 measurements of each day have been grouped into 24 measurements that correspond to the hourly measurements of the measurements vector. These hourly mean vectors have been concatenated chronologically giving a vector composed of $24 \times 16=384$ variables per day. On the set formed by those daily vectors, from which the vectors identified as anomalies by the user have been eliminated, the LOCI anomaly detection method has been applied (Papadimitriou et al., 2003) using Matlab software to obtain a normality model that evaluates the complete set in order to obtain a score that accounts for the degree in which each vector can be identified as an anomaly. Consequently, a threshold on this score has been defined that allows us to ob- tain a classifier that identifies the anomalies against the normal vectors. With this information we can obtain a tagged dataset that has the same number of positive examples (anomalies) as negative ones (normal vectors). Afterward, the ReliefF feature selection method (Kira and Rendell, 1992) can be applied (using Weka software) to obtain an ordering of the variables according to their ability to predict the output class, in this case the presence of an anomaly. That sorted list will be used to select only the most relevant variables and discard the remaining. This is an important contribution taking into account the increasingly high dimension of modern datasets in building energy efficiency.

\subsection{Control Charts for Functional Data}

A methodology to build process control charts for functional data is proposed. The control consists of two phases: Phase I of process calibration and Phase II of process monitoring.

1. PHASE I: A control chart for functional data based on functional data depth and rank control charts is developed. With $\left\{X_{1}(t), X_{2}(t), \ldots, X_{n}(t)\right\}, \quad$ observations of a functional variable $X$, this hypothesis is tested: $H_{0}: X_{i}(t) \stackrel{d}{=} X_{j}(t), \forall i, j \in\{1, \ldots, n\}$, with respect to $H_{a}: X_{i}(t) \stackrel{d}{\neq} X_{j}(t)$, for some $i, j \in\{1, \ldots, n\}$

- An iterative method to detect and discard atypical curves in order to obtain a process sample under control is performed.

- The depth of each curve is calculated with respect to $D\left(X_{i}\right)_{i=1}^{n}$ using functional data depth measures such as Fraiman, mode and random projections.

- The lower control limit (LCL) of rank chart is chosen by a bootstrap procedure based on trimming:

- Reorder the curves according to their depths in a decreasing way. $x_{(1)}, \ldots, x_{(N)}$.

- It is assumed that at most $\alpha \%$ of the sample can be considered atypical data.

- $B$ samples $X_{i}^{* b}, i=1, \ldots, N, b=1, \ldots, B$ are obtained by a smoothed bootstrap:

* A uniform sampling is done, $i^{*}$ of $1, \ldots,[N(1-\alpha)]$.

* $Z_{i *}$ is generated as a Gaussian process with zero mean and variance-covariance matrix $\delta \Sigma_{X}$ with $\delta \in[0,1]$. Where $\Sigma_{X}$ is the variance and covariance matrix of observations $X_{(1)}, \ldots, X_{([N(1-\alpha)])}$.

* Finally, $X_{i}^{* b}=X_{(i *)}+Z_{i *}$ is obtained 
- For each $b=1, \ldots, B$, we obtain $C^{b}$, the $1 \%$ quantile of depth distribution, $D\left(X_{i}^{* b}\right)$. The final value $C=L C L$ is the median of $C^{b}$.

- Curves that verify $D\left(X_{i}\right) \leq L C L$ are outliers and thus process is out of control. They have to be discarded. A chart that includes the original curves and the functional envelope obtained from $99 \%$ of the deeper bootstrap replicas is also performed.

2. PHASE II: Another control chart based on rank control charts and functional depth is proposed to monitor the process.

- We want to monitor the sample $\left\{X_{n+1}(t), X_{n+2}(t), \ldots, X_{m}(t)\right\}$ from $\mathrm{G}$ distribution, taking into account the calibrating sample obtained in Phase I $\left\{X_{1}(t), X_{2}(t), \ldots, X_{n}(t)\right\}$, belonging to $\mathrm{F}$ distribution. The $H_{0}: F=G$ versus $H_{1}: F \neq G$ is tested.

- From $\left\{X_{1}(t), X_{2}(t), \ldots, X_{n}(t)\right\}$, the depths for calibration sample $D\left(X_{i}\right)_{i=1}^{n}$, and for monitored sample, $D\left(X_{j}\right)_{j=n+1}^{m}$, are obtained.

- The rank statistic for monitored sample are estimated by $r_{G}\left(X_{n+1}\right), \ldots, r_{G}\left(X_{m}\right)$, using as reference sample $\left\{X_{1}(t), X_{2}(t), \ldots, X_{n}(t)\right\}: r_{G}(X)=$ $\frac{\#\left\{X_{i} \mid D\left(X_{i}\right) \leq D(X), i=1, \ldots, n\right\}}{n}$

- The rank statistic, the center line $\mathrm{CL}=0.5$, and the LCL $\alpha$ are plotted in a control chart. The process is monitored. IF $r_{G}\left(X_{j}\right) \leq L C I$ for some $j$, the process is out of control. Functional control chart is developed, including original curves and the functional envelop of the $99 \%$ deeper curves of calibrating sample.

\section{RESULTS}

\subsection{Machine Learning Approach for Anomaly Detection and Features Selection}

The calendar image is updated to reflect the anomaly score predicted by the LOCI method (Figure 2). The bar that appears in the background of each panel represents that score. The bar is ochre if it exceeds the first threshold (pre-alarm threshold) and red if it exceeds the second threshold (alarm threshold). The days labeled as anomalies by the maintenance personal have been removed from the training set. Then, the estimated model has been used to classify between normal system performance and anomaly using the complete set. The two used thresholds are those defined by a score equal to 1.5 (which identifies as
Table 1: Variables orderer taking into account their relationship with respecto to the identification of an anomaly.

\begin{tabular}{|c|c|}
\hline Score & Variable \\
\hline 0.045694452 & Indoor temperature (CL02, sale area) \\
0.041626373 & Temperature for general entrance of water \\
0.041031993 & Supply-temperature (CL02, sale area) \\
0.040609546 & Water return temperature (CL02, sale area) \\
0.036097602 & Water return temperature (CL01, sale area) \\
0.035123600 & Supply-temperature (CL01, sale area) \\
0.034024427 & Indoor temperature (CL01, sale area) \\
0.031257874 & Energy consumption of HVAC (KW) \\
0.03001870 & Relative humidity (Sales) \\
0.023807473 & Water return temperature (CL03, store) \\
0.022419010 & Supply-temperature (CL03, store) \\
0.022344179 & Indoor temperature (CL03, store) \\
0.020897972 & Overall energy consumption (KW) \\
\hline
\end{tabular}

anomalies approximately 1 in 4 days) and by a score of 2.5 (which marks as anomaly approximately 1 in 10 days). It is important to note that the Figure 2 only shows the variable of total energy consumption for an illustrative purpose.

The ReliefF method application provides an ordered list of variables according to their importance to identify anomalies from normal performance (taking into account the chosen anomaly level, 1.5 or 2.5). The more influencing variables are those related to the supply and return temperature of HVAC, and even the water temperature of general HVAC system, than energy consumption and indoor temperatures. In addition, those variables corresponding to afternoon and evening are more related with the anomalies than those corresponding to the morning. The assignable cause is that the maximum occupation occurs from 13:00. Therefore, monitoring these features is strongly recommended. Moreover, an average of the weights (importance) of each sensor-meter throughout the day has been performed (see Table 1).

Moreover, once the score variable is obtained, control charts for scalar variables can be applied. A Tukey transformation is previously applied in order to the scores are Gaussian distributed (Tukey, 1977). Figure 3 shows the application of control charts for individual observations (Montgomery, 2007). The natural control limits are estimated using a retrospective sample from August to October 2017. The anomalies corresponding to August, September and October have been identified and removed from the retrospective dataset through an iterative process. Then, the sample corresponding to November has been monitored (compared with respect to the previous calculated natural control limits). We can observe that the process lead out of control at the beginning of November: points (each one corresponding to a different day) fall out of control limits. This is an indicative 


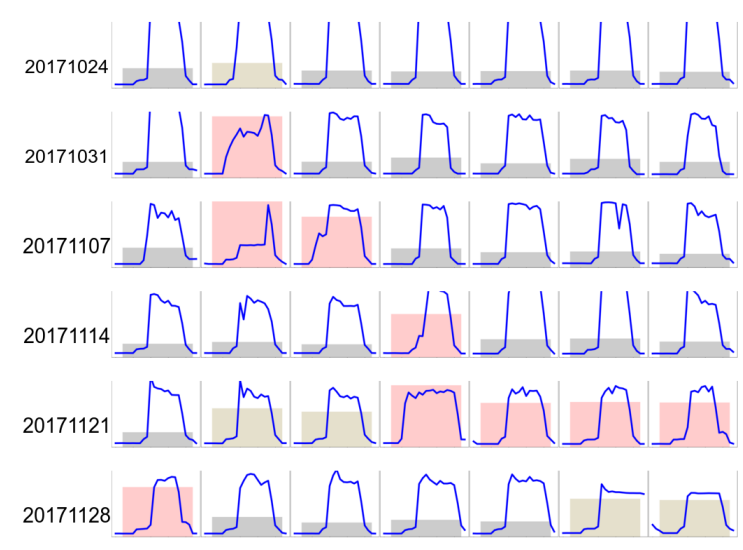

Figure 2: Calendar that shows each day symbolized by its daily energy consumption curve. The height and color of the background bar indicate how plausible the the detection of an anomaly is (ochre color means pre-alarm and red accounts for an alarm).

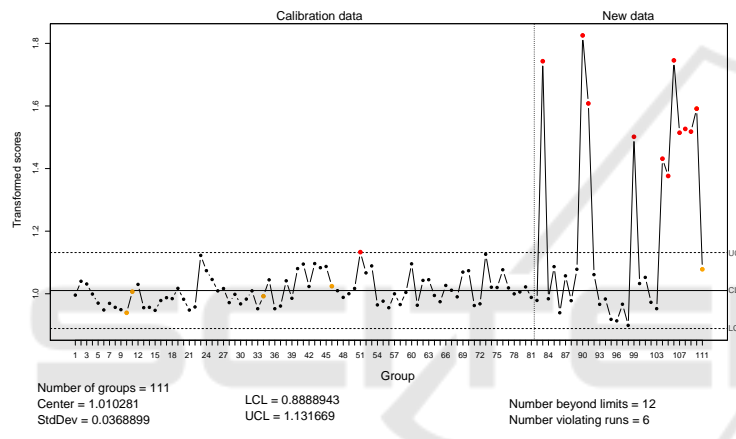

Figure 3: Control chart for individual measurements applied to the score variable obtained by LOCI method. The control limits are obtained using the calibration or retrospective sample.

of something in the installation has changed. The assignable cause is that HVAC installation had been repaired as pointed out in Section 2.

\subsection{Application of FDA Approach for Control Charts}

Phase I control chart for functional data is applied to a retrospective sample of daily HVAC energy consumption that involves the measurements obtained between August and September (Figure 4). All the previously mentioned anomalies (see Section 2) have been detected by the application of functional depth chart during an iterative process. The real curves representation helps to identify the assignable cause of each anomaly. Namely, the absence of energy consumption peak due to improvements during November 2017, changes in opening and closing timetable, and failures in sensors and HVAC installations, among other assignable causes.
A) PHASE I CONTROL CHART: FIRST ITERATION SAMPLE

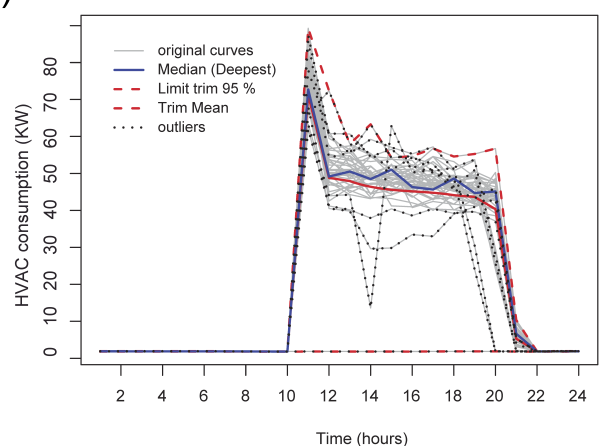

B)

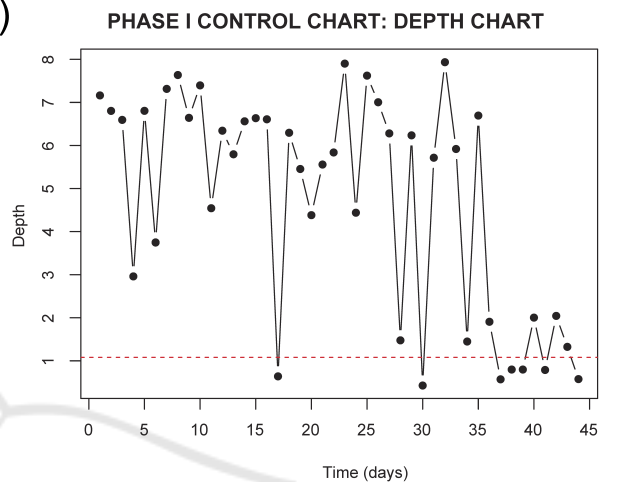

C)

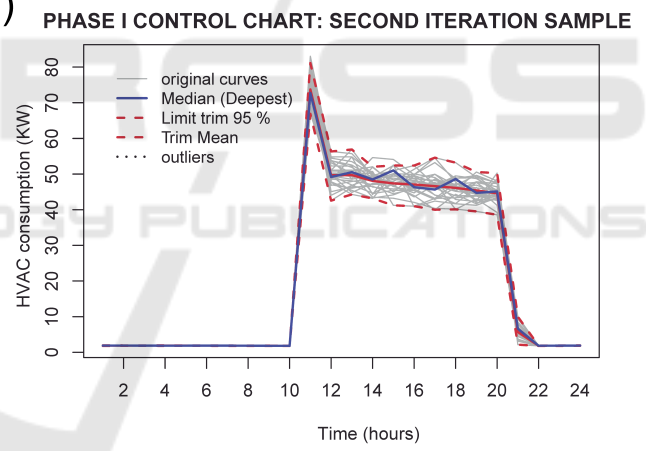

D)

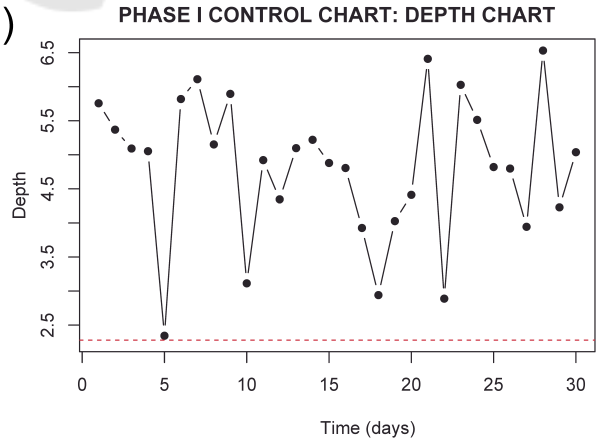

Figure 4: Panel A: Phase I FDA control chart where the first iteration of outlier detection is implemented. Panel B: The corresponding control chart based on FDA data depth (Fraiman and Muniz depth). Panel C: Phase I FDA control chart once the outlier detection is implemented (sample under control). Panel D: Control chart based on FDA data depth once the sample is under control. 
A)

PHASE II CONTROL CHART: RANK CONTROL CHART

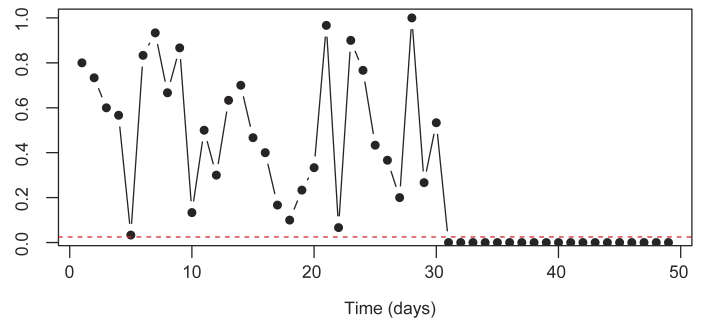

B)

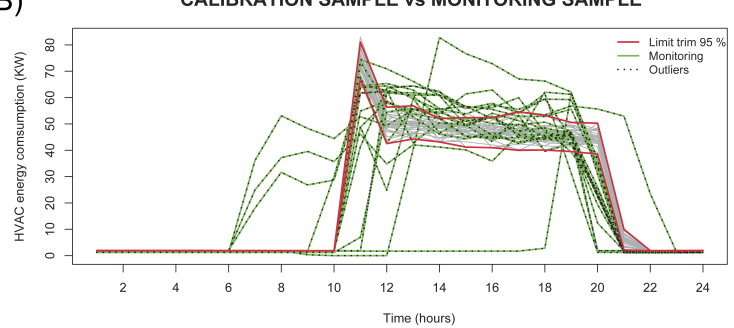

Figure 5: Panel A: Phase II ontrol chart based on FDA data depth (Fraiman and Muniz depth). Panel B: The corresponding Phase II FDA control chart where curves of both calibration and monitoring sample are ploted.

Once the reference sample is obtained, a Phase II control chart based on a nonparametric rank chart with functional depth is applied (Figure 5). The consumption curves corresponding to November are monitored and detected as anomalies when compared with the reference sample.

In order to evaluate the performance of the proposed FDA control chart, a simulation study has been performed. Following other previous works dealing with outlier detection (Febrero et al., 2008), simulated curves have been generated as follows, assuming a Gaussian process, $X(t)=\mu(t)+\sigma(t) \cdot \varepsilon(t)$, where $\sigma^{2}(t)=0.5$ and $\mu(t)=\mathbf{E}(X(t))=30 t(1-$ $t)^{3 / 2}$. Moreover $\varepsilon(t)$ is a Gaussian process $\varepsilon(t) \sim$ $G P(\mathbf{0}, \Sigma)$ with mean $\mathbf{0}$ and variance covariance matrix $\mathbf{E}\left[\varepsilon\left(t_{i}\right) \times \varepsilon\left(t_{j}\right)\right]=e^{-\frac{\left|t_{i}-t_{j}\right|}{0.3}}$.

In order to generate scenarios of atypical curves (varying in mean an shape), the following means are considered taking into account other previous work (Febrero et al., 2008).

- Magnitude change (M1): $\mu(t)=30 t(1-t)^{3 / 2}+\delta$, with $\delta$ control the change of magnitude at levels between 0.4 and 2 .

- Shape change (M2): $\mu(t)=(1-\eta) \cdot 30 t(1-$ $t)^{3 / 2}+\eta \cdot 30 t^{3 / 2}(1-t)$, with $\eta$ the shape change level, between 0.2 and 1 .

Dependent curves are generated by the model $\tilde{Y}_{i}(t)=\mu(t)+\sigma(t) \cdot \tilde{\varepsilon}(t)$, con $\tilde{\varepsilon}(t)=\rho \cdot \tilde{\varepsilon}_{i-1}(t)+(1-$ $\rho) \cdot \varepsilon_{i}(t)$. Whereas $\rho$ is the measure of correlation be- tween curves, $\sigma(t)=0.5$, and $\varepsilon(t)$ and $\tilde{\varepsilon}(t)$ are Gaussian processes.

Table 2 shows the $\hat{p}_{c}$ (proportion of curves correctly detected as anomalies, $\%$ ) and $\hat{p}_{f}$ (proportion of false alarms, \%) for $M_{1}$ and $M_{2}$ cases under the assumption of dependence between curves $(\rho=$ 0.9 ), obtained from $B=1000$ resamples, with $\alpha=$ 0.01 . Fraiman and Muniz (FM), Random projections (RM) and Mode data depth types have been applied (Febrero-Bande et al., 2012). The results supports the application to real study cases.

Table 2: $\hat{p}_{c}$ (proportion of curves correctly detected as anomalies, \%) and $\hat{p}_{f}$ (proportion of false alarms, \%) for $M_{1}$ and $M_{2}$ cases under the assumption of dependence between curves $(\rho=0.9)$, obtained from $B=1000$ resamples, with $\alpha=0.01$.

\begin{tabular}{|c|c|c|c|c|c|c|c|c|c|c|c|c|}
\hline & & $\delta$ & & 4 & 0 & 8 & & .2 & & 1.6 & & 2 \\
\hline Scenario & Size & Depth & $\hat{p}_{f}$ & $\hat{p}_{c}$ & $\hat{p}_{f}$ & $\hat{p}_{c}$ & $\hat{p}_{f}$ & $\hat{p}_{c}$ & $\hat{p}_{f}$ & $\hat{p}_{c}$ & $\hat{\hat{p}_{f}}$ & $\hat{p}_{c}$ \\
\hline \multirow[t]{12}{*}{$M_{1}$} & 50 & FM & 0.45 & \begin{tabular}{|l|l|}
24.60 \\
\end{tabular} & 0.09 & 52.30 & 0.00 & 42.10 & 0.00 & 25.90 & 0.00 & 15.40 \\
\hline & & Mode & $\begin{array}{l}0.93 \\
0.20\end{array}$ & $\begin{array}{l}29.70 \\
45.80\end{array}$ & 0.02 & $\begin{array}{l}60.00 \\
85.30\end{array}$ & $\begin{array}{l}0.07 \\
0.00\end{array}$ & $\begin{array}{l}66.90 \\
94.30\end{array}$ & $\begin{array}{l}0.01 \\
0.00\end{array}$ & $\begin{array}{l}67.30 \\
90.50\end{array}$ & $\begin{array}{l}0.00 \\
0.00\end{array}$ & $\begin{array}{l}65.00 \\
86.30\end{array}$ \\
\hline & 100 & $\mathrm{FM}$ & 1.04 & 31.60 & 0.77 & 83.60 & 0.66 & 98.90 & 0.56 & 100.00 & 0.46 & 100.00 \\
\hline & & & & 32.50 & & 82.30 & & 97.90 & 6 & 99.90 & 126 & 100.00 \\
\hline & & Mode & 0.79 & 43.10 & 0.70 & 91.90 & 0.63 & 99.50 & 0.57 & 100.00 & 0.49 & 100.00 \\
\hline & & $\eta$ & \multicolumn{2}{|c|}{0.2} & \multicolumn{2}{|c|}{0.4} & \multicolumn{2}{|c|}{0.6} & \multicolumn{2}{|c|}{0.8} & \multicolumn{2}{|c|}{1} \\
\hline & 50 & FM & 0.67 & 5.95 & 0.39 & 16.30 & 0.19 & 22.35 & 0.09 & 21.05 & 0.06 & 20.05 \\
\hline & & & 1.24 & 6.95 & 0.95 & 16.75 & 0.59 & 24.95 & 0.36 & & 0.24 & 33.35 \\
\hline & & Mode & 0.21 & 39.90 & 0.04 & 83.80 & 0.00 & 94.90 & 0.00 & 94.70 & 0.00 & 90.90 \\
\hline & 100 & FM & 1.17 & 5.75 & 0.97 & 23.20 & 0.88 & 40.00 & 0.88 & 46.10 & 0.92 & 48.30 \\
\hline & & 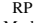 & 169 & 6. & 16 & & 1.65 & & 75 & & 1.82 & \\
\hline & & Mode & 0.81 & 38.40 & 0.77 & 87.90 & 0.73 & 99.80 & 0.72 & 100.00 & 0.69 & 100.00 \\
\hline
\end{tabular}

\section{CONCLUSIONS}

Companies of energy sector need statistical and machine learning tools that allow us to automate the anomaly detection and quality control of energy efficiency in buildings (commercial centers, hospitals, hotels, offices, stores, among others). The real case study of a clothing shop in a mall of Panama City has been provided, for which all the anomalies have been identified by the maintenance staff during a period of one year. Two approaches for anomaly detection and quality control of energy efficiency installations in buildings have been proposed and applied to this case study in order to automate the process. A machine learning methodology for anomaly detection based on the application of LOCI method has been applied. It allows us to define two different levels of anomaly or alarm (pre-alarm and alarm) from the calculation of scores that accounts for relation of each day with respec to the presence of an anomaly. Taking into account these levels, the anomalies previously indicated by the maintenance staff have been successfully identified. In addition, the ReliefF method has been also applied to select which variables are more related with the presence of anomalies. The more critical variables for the quality of the energy efficiency system are the temperatures corresponding to the HVAC installa- 
tion (chiller supply and return temperatures and water temperature). Consequently, the anomaly detection procedure could be applied to these variables, making this process more simple and efficient in terms of time and computation requirements. Alternatively, when we are interested on controlling just one variable and this is characterized by a functional nature, a FDA methodology for control charts has been proposed and applied to the HVAC energy consumption daily curves. Confidence bands have been estimated in the calibration stage, allowing us to monitor the consumption curves of new days and decide if they correspond to anomalies in the system. This statistical approach is based on functional data depth calculation and the application of rank control charts.

\section{ACKNOWLEDGEMENTS}

This research/work of Salvador Naya, Javier TarríoSaavedra and Rubén Fernández-Casal have been supported by MINECO grants MTM2014-52876-R and MTM2017-82724-R, and by the Xunta de Galicia (Grupos de Referencia Competitiva ED431C-2016015 and Centro Singular de Investigación de Galicia ED431G/01 2016-19), all of them through the ERDF. The work of Carlos Erias, Verónica Bolón and Javier Tarrío has been also developed in the framework of eCOAR project (PC18/03) of CITIC. The research of Miguel Flores has been partially supported by Grant PII-DM-002-2016 of Escuela Politécnica Nacional of Ecuador.

\section{REFERENCES}

Barbeito, I., Zaragoza, S., Tarrío-Saavedra, J., and Naya, S. (2017). Assessing thermal comfort and energy efficiency in buildings by statistical quality control for autocorrelated data. Applied energy, 190:1-17.

Bolon-Canedo, V., Sanchez-Maro no, N., and AlonsoBetanzos, A. (2017). Artificial Intelligence: Foundations, Theory, and Algorithms Feature Selection for High-Dimensional Data. Springer.

Bolon-Canedo, V., Sanchez-Marono, N., and AlonsoBetanzos, A. (2011). Feature selection and classification in multiple class datasets: An application to kdd cup 99 dataset. Expert Systems with Applications, 38(5):5947-5957.

Febrero, M., Galeano, P., and González-Manteiga, W. (2008). Outlier detection in functional data by depth measures, with application to identify abnormal nox levels. Environmetrics: The official journal of the International Environmetrics Society, 19(4):331-345.

Febrero-Bande, M., de la Fuente, M. O., et al. (2012). Statistical computing in functional data analysis: The $\mathrm{r}$ package fda. usc. Journal of statistical Software, 51(4):1-28.

Ferraty, F. and Vieu, P. (2006). Nonparametric functional data analysis: theory and practice. Springer Science \& Business Media.

Flores, M., Tarrío-Saavedra, J., Fernández-Casal, R., and Naya, S. (2018). Functional extensions of mandel's $\mathrm{h}$ and $\mathrm{k}$ statistics for outlier detection in interlaboratory studies. Chemometrics and Intelligent Laboratory Systems, 176:134-148.

Francisco-Fernández, M., Tarrío-Saavedra, J., Mallik, A., and Naya, S. (2012). A comprehensive classification of wood from thermogravimetric curves. Chemometrics and Intelligent Laboratory Systems, 118:159_ 172.

Kira, K. and Rendell, L. A. (1992). A practical approach to feature selection. In Machine Learning Proceedings 1992, pages 249-256. Elsevier.

Lee, J., Kao, H.-A., and Yang, S. (2014). Service innovation and smart analytics for industry 4.0 and big data environment. Procedia Cirp, 16:3-8.

Liu, R. Y. (1995). Control charts for multivariate processes. Journal of the American Statistical Association, 90(432):1380-1387.

López-Pintado, S. and Romo, J. (2009). On the concept of depth for functional data. Journal of the American Statistical Association, 104(486):718-734.

Mallik, A., Tarrío-Saavedra, J., Francisco-Fernández, M., and Naya, S. (2011). Classification of wood micrographs by image segmentation. Chemometrics and intelligent laboratory systems, 107(2):351-362.

Montgomery, D. C. (2007). Introduction to statistical quality control. John Wiley \& Sons.

Naya, S. (2017). Industry 4.0. an opportunity for the relationship between university and shipbuilding in the future. In Pan-American Conference of Naval Engineering, pages 169-177. Springer.

Papadimitriou, S., Kitagawa, H., Gibbons, P. B., and Faloutsos, C. (2003). Loci: Fast outlier detection using the local correlation integral. In Proceedings 19th International Conference on Data Engineering (Cat. No. 03CH37405), pages 315-326. IEEE.

Tukey, J. W. (1977). Exploratory data analysis.

Woodall, W. H. (2007). Current research on profile monitoring. Production, 17(3):420-425. 